\title{
ErbB4 promotes cyclooxygenase-2 expression and cell survival in colon epithelial cells
}

\author{
Mark R Frey ${ }^{1}$, Valda C Hilliard ${ }^{2}$, Matthew T Mullane ${ }^{1}$ and D Brent Polk ${ }^{1,2}$
}

The ErbB4 receptor tyrosine kinase is expressed at high levels in human and mouse colitis, and inhibits colon epithelial cell apoptosis in the presence of proinflammatory cytokines. In this study, we investigated the molecular mechanisms responsible for ErbB4-induced cell survival. In cultured mouse colon epithelial cells, ErbB4 overexpression resulted in increased levels of cyclooxygenase-2 (COX-2) mRNA and protein; in contrast, ErbB4 knockdown with siRNA blocked COX-2 accumulation in response to tumor necrosis factor. Although ErbB4 is expressed as up to four isoforms in epithelial tissues, its ability to promote COX-2 expression was isoform independent. ErbB4-stimulated COX-2 induction was associated with an increase in mRNA half-life and was blocked by inhibition of Src, phosphatidylinositol (PI) 3-kinase, or epidermal growth factor receptor (EGFR). Furthermore, ErbB4 expression promoted EGFR phosphorylation in the presence of heregulin, implicating ErbB4-EGFR heterodimerization in these responses. As to the cellular responses to ErbB4 activation, increased survival of ErbB4-expressing cells in the presence of proinflammatory cytokines was sensitive to the COX-2 inhibitor celecoxib. Furthermore, ErbB4-overexpressing cells acquired the ability to form colonies in soft agar, indicative of cellular transformation, also in a celecoxib-sensitive manner. Together our data indicate that ErbB4 is a key regulator of COX-2 expression and cellular survival in colon epithelial cells, acting in concert with EGFR through a Src- and PI 3-kinase-dependent mechanism. These results suggest that chronic overexpression of ErbB4 in the context of inflammation could contribute to colitis-associated tumorigenesis by inhibiting colonocyte apoptosis.

Laboratory Investigation (2010) 90, 1415-1424; doi:10.1038/labinvest.2010.117; published online 28 June 2010

KEYWORDS: cell survival; colon epithelial cells; cyclooxygenase-2; ErbB4; inflammatory bowel diseases; receptor tyrosine kinases

The ErbB4 receptor tyrosine kinase is a member of the epidermal growth factor receptor (EGFR)-related ErbB family, along with EGFR/ErbB1, HER2/ErbB2, and HER3/ErbB3. It is broadly expressed in fetal and adult mammalian tissues, ${ }^{1}$ and following ligand-binding becomes active either as homodimers, heterodimers with other ErbBs, or as a constitutively active intracellular domain proteolytic cleavage product. $^{2}$ ErbB4 expression and activity have been linked to a variety of cellular processes including differentiation, ${ }^{3,4}$ cell survival, ${ }^{5}$ migration, ${ }^{6}$ proliferation, ${ }^{3,7}$ growth arrest ${ }^{8}$ and tumorigenesis ${ }^{9,10}$ in different tissues. However, only limited data are available on the intracellular signaling pathways involved in these responses. ErbB4 has several unusual biochemical properties - the ability to bind both heregulin/ neuregulin growth factors and a subset of EGFR ligands, ${ }^{11}$ the expression and metalloproteinase processing of up to four alternatively spliced isoforms, ${ }^{6,8}$ and association with a more restricted suite of $\mathrm{SH} 2$-containing targets than ErbB1-3 $3^{12}-$ which make it unique both in terms of its signaling and its possible role in disease. Further characterization of the molecular pathways downstream of ErbB4 activation will be key to advancing understanding of the receptor's biology.

ErbB4 is expressed at elevated levels in the inflamed colonic mucosa of Crohn's disease patients, is induced in cultured cells by the potent inflammatory cytokine tumor necrosis factor (TNF), and promotes survival of cultured colon epithelial cells in a phosphatidylinositol (PI) 3-kinasedependent manner. ${ }^{13}$ Furthermore, ErbB4 deletion from colorectal cancer cells promotes apoptosis. ${ }^{14}$ Together, these observations suggest that ErbB4 is a key regulator of colonocyte survival in inflammation and tumorigenesis, though the underlying signaling pathways remain undetermined.

Cyclooxygenase-2 (COX-2), the inducible form of the mammalian prostaglandin synthase, catalyzes the production

\footnotetext{
${ }^{1}$ Division of Gastroenterology, Hepatology and Nutrition, Department of Pediatrics, Vanderbilt University School of Medicine, Nashville, TN, USA and ${ }^{2}$ Department of Cell and Developmental Biology, Vanderbilt University School of Medicine, Nashville, TN, USA

Correspondence: Dr MR Frey, PhD, Division of Gastroenterology, Hepatology and Nutrition, Department of Pediatrics, Vanderbilt University School of Medicine, 2215 Garland Avenue, MRB IV 1055B, Nashville, TN 37232-0696, USA.

E-mail: mark.frey@vanderbilt.edu

Received 2 February 2010; revised 16 April 2010; accepted 30 April 2010
} 
of prostaglandins from arachidonic acid, simultaneously modulating inflammatory responses ${ }^{15}$ and promoting intestinal epithelial cell survival. ${ }^{16,17}$ COX-2 is present at high levels during inflammation and the response to injury in a number of tissues, including the digestive tract. ${ }^{18}$ It has also been identified as a key participant in colorectal carcinogenesis. ${ }^{19,20}$ A better understanding of the cellular mechanisms regulating COX-2 expression and activity is likely to open new therapeutic avenues for both inflammatory diseases and cancer.

In this study, we tested the hypothesis that COX-2 is a target of ErbB4 signaling. We report that ErbB4 overexpression in mouse colon epithelial cells resulted in elevated COX-2 expression. This effect was associated with increased COX-2 mRNA half-life and was blocked by EGFR, Src, or PI 3-kinase inhibition. In addition, ErbB4 expression in these cells promoted cell survival and anchorage-independent growth, which were sensitive to the COX-2 inhibitor celecoxib, and ErbB4 knockdown from colon cancer cells resulted in decreased COX-2 expression and increased sensitivity to TNF-induced cell death. Together these results suggest that ErbB4 promotes colonocyte survival through COX-2 expression, and that this pathway may represent a useful therapeutic target for inflammatory disorders and cancer.

\section{MATERIALS AND METHODS Cell Culture}

The nontransformed, conditionally immortalized young adult mouse colon (YAMC) epithelial cell line was provided by $\mathrm{Dr}$ Robert Whitehead and the Vanderbilt Digestive Diseases Research Center Novel Cell Line Core. ${ }^{21}$ These cells express a temperature-sensitive SV40 Large $\mathrm{T}$ antigen that confers conditional immortalization, but not transformation, under permissive conditions $\left(33^{\circ} \mathrm{C}\right.$ in RPMI 1640 with $5 \%$ FBS, $5 \mathrm{U} / \mathrm{ml}$ mouse interferon- $\gamma$ (IFN- $\gamma$; Intergen, Norcross, GA, USA), $100 \mathrm{U} / \mathrm{ml}$ penicillin and streptomycin, $5 \mu \mathrm{g} / \mathrm{ml}$ insulin, $5 \mu \mathrm{g} / \mathrm{ml}$ transferrin, and $5 \mathrm{ng} / \mathrm{ml}$ selenous acid (BD Biosciences, San Jose, CA, USA)). YAMC cells express low but detectable levels of ErbB4 in the resting state. ${ }^{13}$ Parental YAMC and ErbB4-infected YAMC cell lines were maintained under permissive conditions and transferred to nonpermissive conditions (RPMI 1640 containing 0.5\% FBS, streptomycin and penicillin without IFN- $\gamma$, insulin, transferrin, or selenous acid, at $37^{\circ} \mathrm{C}$ ) for $24 \mathrm{~h}$ before signaling experiments. LIM 2405 human colon cancer cells were also provided by Dr Whitehead ${ }^{22}$ and were grown in RPMI 1640 together with 5\% FBS, $100 \mathrm{U} / \mathrm{ml}$ penicillin and streptomycin, $5 \mu \mathrm{g} / \mathrm{ml}$ insulin, $5 \mu \mathrm{g} / \mathrm{ml}$ transferrin, and $5 \mathrm{ng} / \mathrm{ml}$ selenous acid.

\section{Transfections and Constructs}

pCDNA3.1-ErbB4 (JM-a/CYT-1, JM-a/CYT-2, JM-b/CYT-1, and JM-b/CYT-2 isoforms) expression vectors were kindly provided by Graham Carpenter (Vanderbilt University). Inserts from these constructs were PCR-amplified (primers:
5'-ATGGCGATCGCATGAAGCCGGCGACAGGACTTTG-3', Sgfl site; 5'-TTGGGCCGGACCGGCCTTACACCACAGTATT CCGGTG-3', SfiI site) then cut with $S f i$ I and $S g f$ I and ligated into linearized bicistronic LZRS-GFP vector (Albert Reynolds, Vanderbilt University). Recombinant plasmids were screened by SfiI/Sgfl digestion. Phoenix packaging cells (Steve Hanks, Vanderbilt University) were transiently transfected with LZRS-GFP or LZRS-ErbB4-GFP and YAMC cells were subjected to five rounds of infection with filtered supernatant supplemented with $4 \mu \mathrm{g} / \mathrm{ml}$ polybrene. Infected populations were expanded and GFP-positive cells were sorted at the Vanderbilt Medical Center Flow Cytometry Shared Resource using a Becton-Dickinson FACSAria; top 20\% GFP-expressing cells were maintained as pools.

Nontargeting control and ErbB4-specific siRNA pools were purchased from Dharmacon (Lafayette, CO, USA) and transfected into YAMC cells ( $100 \mathrm{nM}$ siRNA) with Lipofectamine RNAiMax (Invitrogen, Carlsbad, CA, USA) following the manufacturer's protocol. Control and ErbB4-targeting lentiviral shRNA particles were purchased from Santa Cruz Biotechnology (Santa Cruz, CA, USA) and introduced to LIM 2405 cells according to the manufacturer's instructions.

\section{Soft Agar Colony Formation}

YAMC cell pools expressing LZRS-GFP (YAMC-Vec) and cells expressing LZRS-ErbB4 (JM-b/CYT-2)-GFP (YAMCErbB4) were embedded in a $0.35 \%$ Noble agar (Sigma Corp., St Louis, MO)/growth medium gel overlaid on a $0.5 \%$ agar/ growth medium support. The ErbB4 ligand heregulin-1 $\beta$ (HRG; $100 \mathrm{ng} / \mathrm{ml})$ or the COX-2 inhibitor celecoxib $(5 \mu \mathrm{M}$; Ray DuBois, Vanderbilt University) were added to both the overlay and supporting gels in some wells. Cultures were maintained under permissive conditions, to model cooperation of ectopic ErbB4 with the SV40 Large T antigen expressed in these cells, for 3 weeks. After 3 weeks in culture at permissive temperature, colonies were stained with MTT and counted by an investigator masked to experimental conditions.

\section{Antibodies, Cytokines, and Growth Factors}

Antibodies were purchased from: polyclonal anti-ErbB4 (c-18), monoclonal anti-HuR, monoclonal anti-CUGBP-2, and goat polyclonal anti-COX-2 (Santa Cruz Biotechnology); monoclonal anti-RBM3 (Novus Biologicals, Littleton, CO, USA); monoclonal anti-actin (Sigma); anti-phospho-Y1284 ErbB4, phospho-Y1068 EGFR, phospho-S536 p65 NF- $\kappa$ B and HRP-conjugated secondary antibodies (Cell Signaling, Danvers, MA, USA). Recombinant HRG was purchased from R\&D Systems (Minneapolis, MN, USA). Murine TNF was purchased from Peprotech (Rocky Hill, NJ, USA). All HRG and TNF cell treatments were at $100 \mathrm{ng} / \mathrm{ml}$.

\section{Cell Lysates, Immunoprecipitation, and Western Blot Analysis}

Cellular proteins were extracted in $50 \mathrm{mM}$ Tris $(\mathrm{pH}$ 7.4) containing $150 \mathrm{mM} \mathrm{NaCl}, 1 \mathrm{mM}$ EDTA, $1 \mathrm{mM}$ EGTA, $1 \%$ 
Triton X-100, $0.2 \%$ sodium deoxycholate, $0.1 \%$ SDS, and $0.1 \%$ protease and phosphatase inhibitor cocktails (Sigma Corp.) and cleared by centrifugation. Whole-cell lysates were immediately subjected to protein assay and boiled in Laemmli loading buffer. For immunoprecipitation, we precleared $1 \mathrm{mg}$ lysate and then incubated with $2 \mu \mathrm{g}$ antibody for $1 \mathrm{~h}$ at $4^{\circ} \mathrm{C}$ followed by $1 \mathrm{~h}$ at $4{ }^{\circ} \mathrm{C}$ with protein $\mathrm{A} / \mathrm{G}$-agarose beads (Santa Cruz Biotechnology). Immunocomplexes were washed $4 \times$ in lysis buffer and eluted by boiling in Laemmli buffer. Samples were separated on SDS-polyacrylamide gels (6-10\% as appropriate) and blotted on nitrocellulose membranes using the manufacturer's instructions for each antibody. Loading was monitored by western blot for actin and at least one additional uninvolved protein for whole-cell lysates.

\section{RNA Isolation and Analysis}

Total RNA was purified with RNeasy columns (Qiagen, Valencia, CA, USA) including on-column DNase treatment. COX-2 mRNA expression was determined by real-time quantitative PCR (RT-qPCR) using an independent cDNA synthesis step with iScript (Bio-Rad, Hercules, CA, USA), SYBR Green reaction mix (Sigma), and iCycler with IQ5 software (Bio-Rad). Relative mRNA levels were determined using the $2^{-\Delta \Delta C T}$ method with $18 \mathrm{~S}$ RNA as the reference. Primers used were: $5^{\prime}$-CGTCTGCCCTATCAACTTTCG (18s forward); 5'-CCTTCCTTGGATGTGGTAGCC (18s reverse); 5'-CTCCCTGAAGCCGTACACAT (COX-2 forward); ATGGT GCTCCAAGCTCTACC (COX-2 rev).

\section{Cytotoxicity Assays}

Cells were plated in 96-well dishes (10000 per well), grown for $24 \mathrm{~h}$, shifted to nonpermissive conditions in the presence of $150 \mathrm{U} / \mathrm{ml} \mathrm{IFN}-\gamma$ for $24 \mathrm{~h}$, then exposed to $100 \mathrm{ng} / \mathrm{ml}$ TNF for $24 \mathrm{~h}$. Cells were counted using an MTS-based colorimetric proliferation assay kit (Promega Corp.) and percentage of cell kill was calculated relative to untreated control wells. Reported values reflect averages of at least four replicate wells.

\section{Statistics and Replicates}

All data are representative of at least three independent experiments. Statistical analyses and mRNA half-life calculations were performed using Prism software (GraphPad Inc., La Jolla, CA, USA). Statistical significance of differences from controls was assessed by ANOVA with Tukey's post-test. Error bars indicate standard error of means.

\section{RESULTS \\ Full-Length ErbB4 Increases COX-2 Levels in Colon Epithelial Cells}

In colonocytes, ErbB4 is induced by, and promotes cell survival in the presence of, inflammatory cytokines such as TNF. $^{13}$ To investigate the mechanisms of this cellular response, we screened ErbB4-overexpressing cells for changes in expression of molecules associated with both cell survival and inflammation. An intriguing target identified was COX-2, which is involved in inflammation, cell survival, and colon carcinogenesis. ${ }^{23,24}$ Western blot analysis of YAMC mouse colon epithelial cells infected with empty vector or retroviral expression constructs for each of the four ErbB4 isoforms (JM-a/CYT-1, JM-a/CYT-2, JM-b/CYT-1, and JM-b/CYT-2) showed increased steady-state levels of COX-2 protein (Figure 1a). Interestingly, this effect did not depend on a particular ErbB4 isoform, but seemed to parallel levels of full-length receptor expressed. Subsequent experiments using ErbB4 overexpression compared cells expressing vector (termed YAMC-Vec below) with cells expressing the 'minimal' isoform JM-b/CYT-2 ErbB4 (termed YAMC-ErbB4 below). COX-2 expression was further enhanced by addition of the ErbB4 ligand HRG for $3 \mathrm{~h}$ (Figure 1b). Elevated COX-2 was also detected in MDCK cocker spaniel kidney epithelial cells overexpressing ErbB4 (Figure 1c), showing that this effect is not specific to the YAMC cell line or the colon. COX-2 activity is generally controlled through changes in message and protein expression, but regulatory phosphorylation on the enzyme has been reported. ${ }^{25}$ Thus we immunoprecipitated COX-2 from YAMC and YAMC-ErbB4 cells with
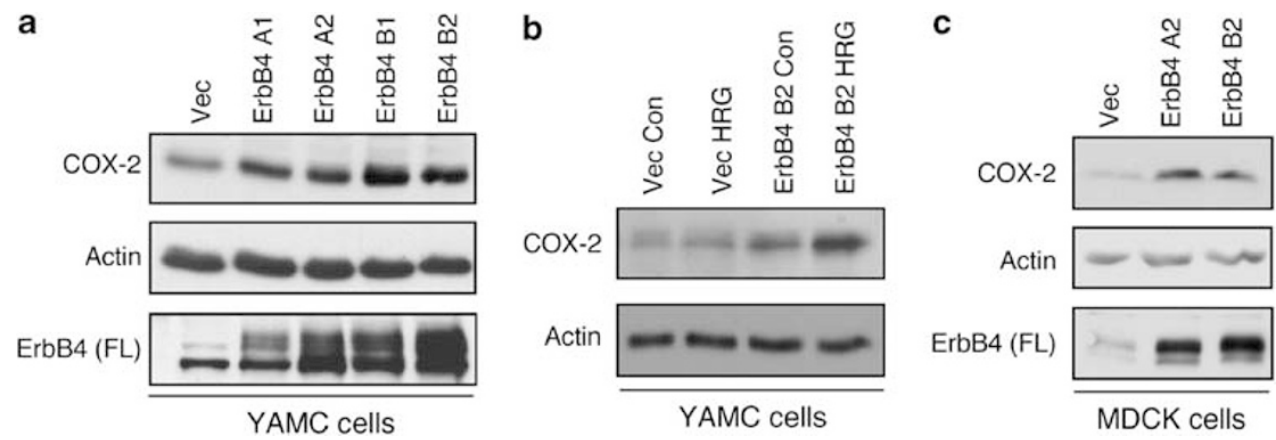

Figure 1 ErbB4 stimulates COX-2 expression in colon epithelial cells. (a) YAMC cells were infected with retroviral vectors expressing each ErbB4 isoform (A1, JM-a/CYT-1; A2, JM-a/CYT-2; B1, JM-b/CYT-1; B2, JM-b/CYT-2). Whole-cell lysates were prepared and subjected to western blot analysis for full-length (FL) ErbB4 and COX-2 expression. Actin blot included as loading control. (b) JM-b/CYT-2-expressing cells (YAMC-ErbB4) were exposed to HRG (100 ng/ml) for $3 \mathrm{~h}$; COX-2 protein levels were determined by immunoblot analysis. (c) MDCK cells were infected with vector or ErbB4-expressing constructs and COX-2 levels were determined by western blot analysis. 

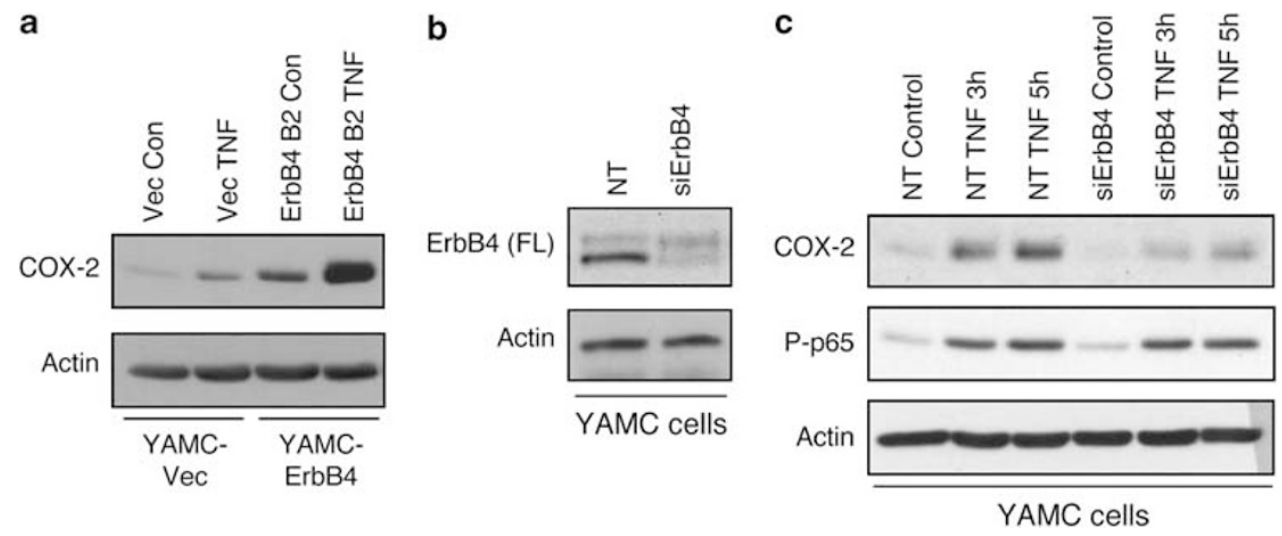

Figure 2 ErbB4 enhances TNF-induced COX-2 expression. (a) YAMC-ErbB4 cells were exposed to TNF (100 ng/ml) for $3 \mathrm{~h}$; COX-2 protein levels were determined by immunoblot analysis. (b) YAMC cells were transfected with nontargeting or ErbB4-specific siRNA pools for $72 \mathrm{~h}$. (c) siRNA-transfected cells were stimulated with TNF; whole-cell lysates were prepared and subjected to immunoblot analysis for COX-2 induction as well as phosphorylation of the p65 NF- $\kappa$ B subunit as control for active TNF signaling.

or without HRG treatment, and blotted with antibodies to phospho-tyrosine, -threonine, and -serine. However, no ErbB4- or HRG-induced phosphorylation on COX-2 was detected (data not shown).

\section{ErbB4 Promotes TNF-Stimulated COX-2 Accumulation} Both ErbB $4^{13}$ and $\mathrm{COX}-2^{23}$ are induced in colon epithelial cells by the proinflammatory cytokine TNF. To test whether these pathways are linked, we treated YAMC-Vec and YAMCErbB4 cells with TNF $(100 \mathrm{ng} / \mathrm{ml})$ and determined COX-2 expression by western blot analysis. TNF induced COX-2 accumulation by $3 \mathrm{~h}$ in YAMC-Vec cells; this response was significantly enhanced by ErbB4 overexpression (Figure 2a). As noted above, these results use the YAMC-ErbB4 cells expressing the JM-b/CYT-2 isoform; similar outcomes were observed with lines expressing other ErbB4 isoforms (not shown), further supporting the conclusion that ErbB4mediated COX-2 induction does not require specific JM or CYT sequences.

Unchallenged YAMC cells express low but detectable basal levels of ErbB4, which increase following cell exposure to TNF. We have previously used siRNA knockdown to show that this endogenous ErbB4 expression regulates acute TNF activation of PI 3-kinase/Akt signaling. ${ }^{13}$ To test the requirement for endogenous ErbB4 in COX-2 regulation by TNF, we transfected YAMC cells with nontargeting or ErbB4specific siRNA pools. As reported in our previous studies, we obtained $>85 \%$ knockdown of endogenous ErbB4 with siRNA transfection (Figure 2b). Following TNF treatment, whole-cell protein lysates were prepared and COX-2 induction was assessed by immunoblot analysis. TNF-induced COX-2 expression was significantly attenuated by ErbB4 knockdown (Figure 2c). Other TNF-induced signaling targets, including phosphorylation of MAP kinases ${ }^{13}$ and the p65 subunit of NF- $\kappa \mathrm{B}$, were not sensitive to ErbB4 deletion.

\section{ErbB4 Enhancement of COX-2 Levels in YAMC Cells is Src and PI 3-Kinase Dependent}

To investigate the molecular pathways involved in ErbB4-stimulated COX-2 expression, we exposed YAMC-ErbB4 cells to HRG or TNF for $3 \mathrm{~h}$ in the presence of Src (CGP77675, $1 \mu \mathrm{M}$ ), PI 3-kinase (LY294002, $5 \mu \mathrm{M}$ ), and MEK (U0126, $10 \mu \mathrm{M}$ ) inhibitors. Blockade of Src or PI 3-kinase, but not MEK, signaling abrogated COX-2 induction in these cells (Figure $3 \mathrm{a}$ and $\mathrm{b}$ ). Results were confirmed with the use of additional Src and PI 3kinase inhibitors (PP2 and wortmannin, respectively; data not shown). Interestingly, Src inhibition also blocked Akt phosphorylation in response to $3 \mathrm{~h}$ HRG exposure (Figure 3a), suggesting that Src is upstream of PI 3-kinase/Akt in ErbB4induced signaling. To further explore this possible relationship, we tested Akt phosphorylation after acute $(5 \mathrm{~min})$ HRG treatment. Src inhibition abrogated HRG-stimulated Akt phosphorylation at this early time point (Figure 3c), supporting the notion that Src is required for activation of the PI 3-kinase pathway by ErbB4 in colon epithelial cells.

\section{ErbB4 Enhancement of COX-2 Levels Requires EGFR}

Treatment with EGF also promotes COX-2 expression levels in YAMC cells (Hobbs and Polk, unpublished observations), raising the possibility that ErbB4 enhances COX-2 by heterodimerization with, or transactivation of, EGFR. Therefore we exposed YAMC-ErbB4 cells to the EGFR inhibitor AG1478 (150 nM, 30 min pretreatment) before TNF or HRG treatment. Whole-cell lysates were prepared and COX-2 levels were assessed by western blot analysis. EGFR inhibition completely blocked both TNF- and HRG-stimulated COX-2 induction in YAMC-ErbB4 cells (Figure 4a). Similarly, transfection with EGFR-specific siRNA abrogated COX-2 induction in the context of ErbB4 overexpression (Figure 4b). Furthermore, siRNA knockdown of EGFR expression attenuated ErbB4 phosphorylation in response to HRG (Figure 4c), suggesting a role for EGFR in ligand-induced 
a
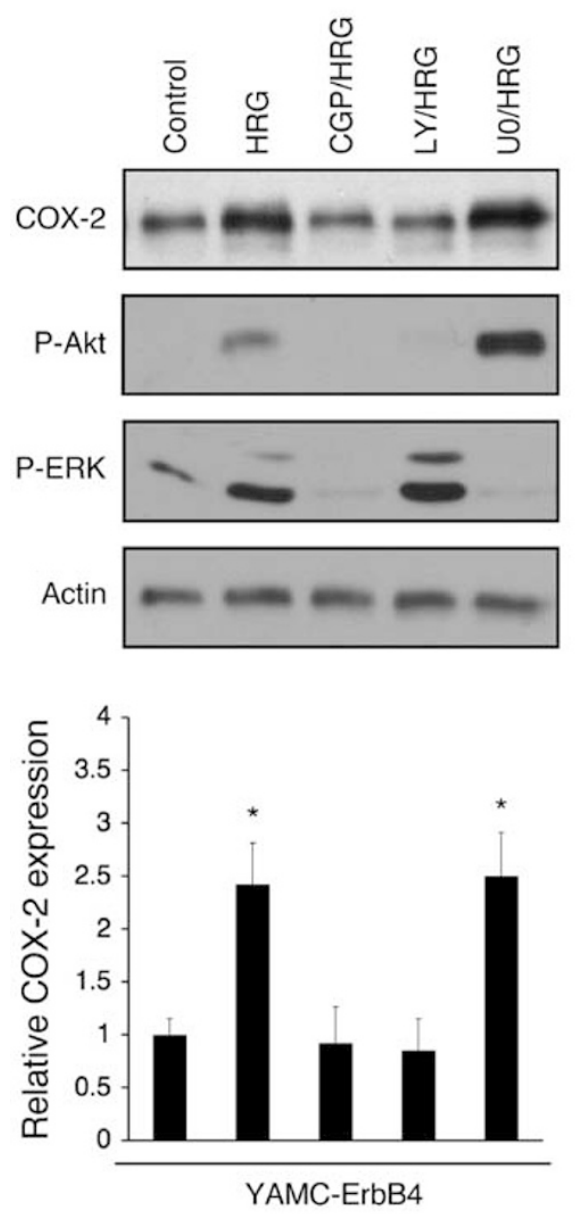

b

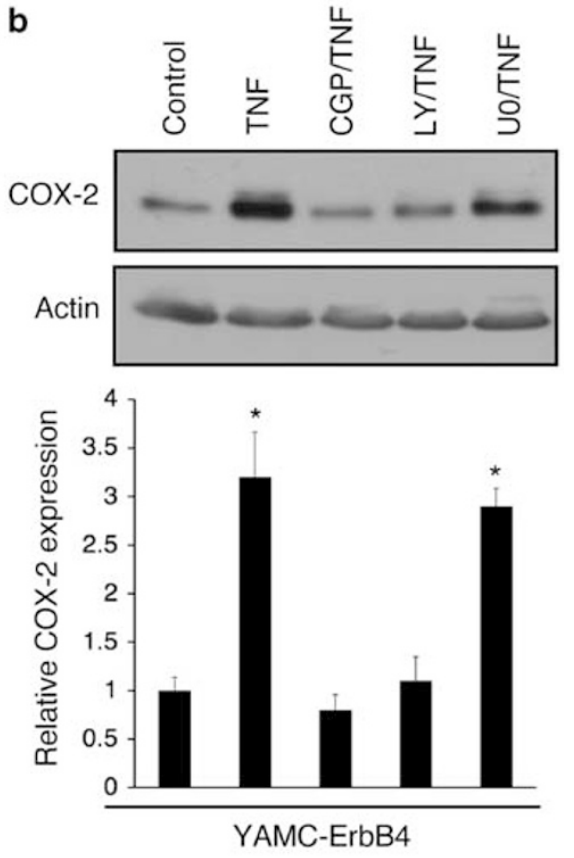

C

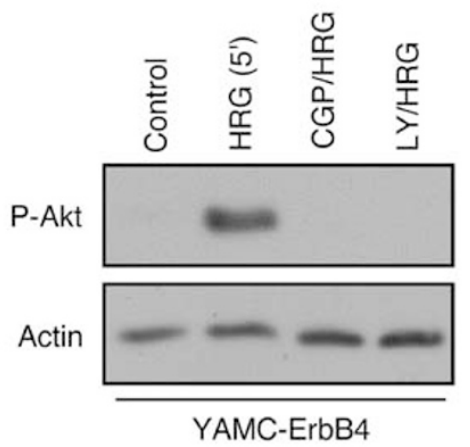

Figure 3 Src and PI 3-kinase activities are required for COX-2 induction in YAMC-ErbB4 cells. YAMC-ErbB4 cells were exposed to CGP77675 (Src inhibitor, $1 \mu \mathrm{M}$ ), LY294002 (PI 3-kinase inhibitor, $5 \mu \mathrm{M}$ ), or U0126 (MEK inhibitor, $10 \mu \mathrm{M}$ ) for 30 min before incubation with either (a) TNF or (b) HRG for $3 \mathrm{~h}$. COX-2 levels were determined by immunoblot analysis. Graphs show densitometry for four experiments. ${ }^{\star} P<0.01$ vs control. (c) YAMC-ErbB4 cells were exposed to HRG for 5 min with or without CGP77675 or LY294002 pretreatment. Akt phosphorylation was determined by western blot analysis.

ErbB4 activation. Using antibodies specific for EGFR phosphorylated on Y1068, we also observed EGFR phosphorylation by $5 \mathrm{~min}$ in ErbB4-overexpressing cells (Figure 4d), paralleling the onset of ErbB4 phosphorylation (note that phosphorylation of the low levels of endogenous ErbB4 were also detectable in YAMC-Vec cells after HRG exposure, albeit only at longer blot exposure times (not shown)). In contrast, in vector-expressing YAMC cells HRG had minimal effect on EGFR phosphorylation/activation at any time point studied. Thus, although EGFR does not bind HRG directly, ${ }^{26}$ it can be activated by this ligand in the presence of ErbB4 and is required for maximal ligand-driven ErbB4 phosphorylation and COX-2 induction.

\section{COX-2 mRNA Levels are Elevated and Message Half-life Extended in ErbB4-Expressing Cells}

COX-2 expression in the mammalian cell is controlled at multiple levels. To investigate the point at which ErbB4 signaling is involved in this regulation, we treated cells with inhibitors of protein translation and RNA synthesis. A $5 \mathrm{~h}$ exposure of YAMC-Vec and YAMC-ErbB4 cells to the translation inhibitor cycloheximide had no appreciable effect on basal COX-2 levels (data not shown). Given that ErbB4 expression enhances COX-2 accumulation in as little as $3 \mathrm{~h}$ following exposure to a stimulus (Figure $1 \mathrm{~b}$ and e) regulation of protein stability/turnover is therefore unlikely to account for observed differences.

In contrast, preincubation with $10 \mu \mathrm{g} / \mathrm{ml}$ actinomycin D to stop RNA synthesis completely blocked HRG- and TNFstimulated COX-2 protein expression in YAMC-ErbB4 cells (Figure 5a), suggesting regulation of mRNA, either at the level of transcription or message stability. RT-qPCR analysis of isolated RNA confirmed an effect on RNA levels; YAMCErbB4 cells showed elevated basal COX-2 steady-state mRNA $v s$ YAMC-Vec (Figure 5b), and an enhanced mRNA accumulation in response to either HRG or TNF. Furthermore, 
a

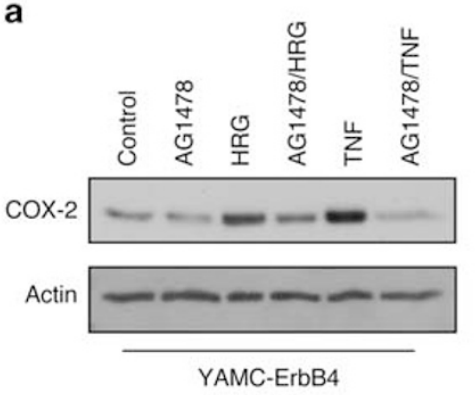

b

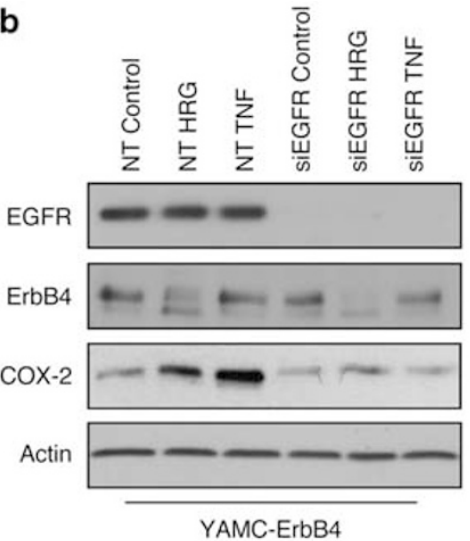

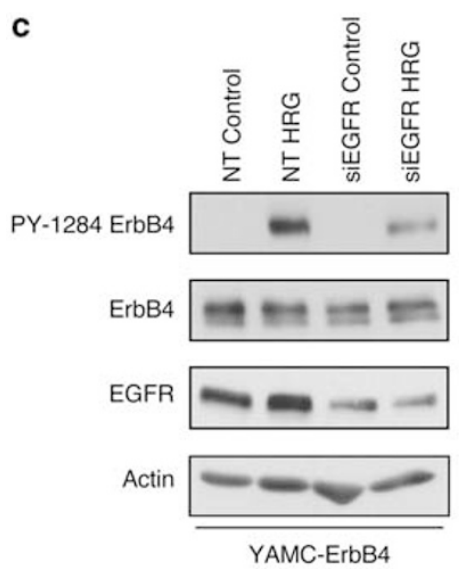

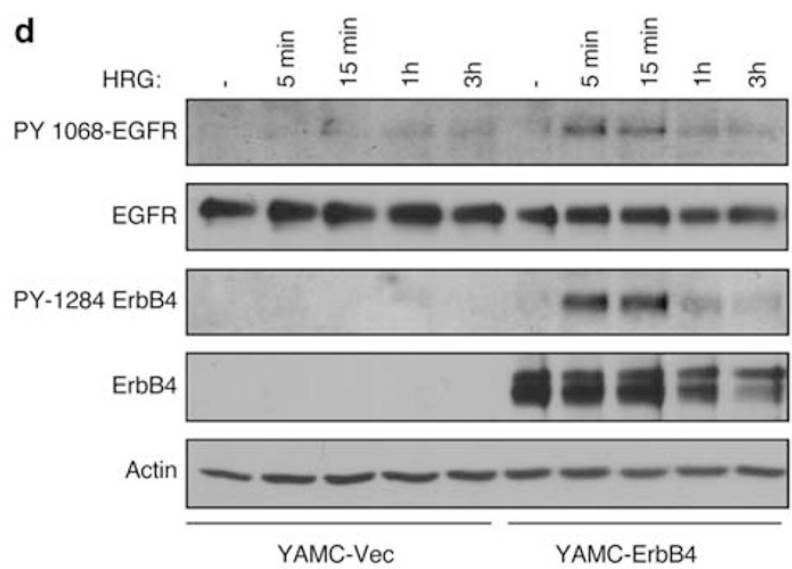

Figure 4 EGFR regulates ErbB4 activation and COX-2 expression. (a) YAMC-ErbB4 cells were incubated with the EGFR inhibitor AG1478 (150 nM) for $30 \mathrm{~min}$ before $3 \mathrm{~h}$ treatment with TNF or HRG. COX-2 levels were determined by western blot analysis. (b) YAMC-ErbB4 cells were transfected with nontargeting or EGFR-specific siRNA pools for $72 \mathrm{~h}$, then stimulated with TNF or HRG. EGFR, ErbB4 and COX-2 levels were determined by immunoblot analysis. (c) YAMC-ErbB4 cells were transfected with nontargeting or EGFR-specific siRNA pools, then stimulated with HRG for 15 min. ErbB4 phosphorylation was determined by western blot analysis. (d) YAMC-Vec and YAMC-ErbB4 cells were exposed to HRG for indicated times; EGFR and ErbB4 phosphorylation were determined by western blot analysis.

siRNA knockdown of ErbB4 from YAMC cells blunted COX-2 mRNA accumulation in response to TNF (Figure 5c), as it did with protein accumulation (Figure $2 \mathrm{~b}$ ). To test whether these responses might reflect a change in COX-2 message half-life, we subjected YAMC-Vec and YAMC-ErbB4 cell cultures to timed incubations with actinomycin $\mathrm{D}$ followed by RT-qPCR of isolated RNA and regression analysis. This analysis showed that the half-life of COX-2 mRNA is extended from $0.87 \mathrm{~h}$ in YAMC-Vec cells to $2.79 \mathrm{~h}$ in YAMCErbB4 cells (Figure 5d). In addition, western blot analysis for CUGBP-2, HuR, and RBM3, proteins that regulate COX-2 message stability, ${ }^{27-29}$ revealed a modest increase in RBM3, but no change in CUGBP-2 or HuR, in YAMC-ErbB4 cells vs YAMC-Vec (Figure 5e). No apparent change in subcellular localization of these regulatory proteins with ErbB4 expression was observed by immunofluorescence localization analysis (data not shown); in both YAMC-Vec and YAMC-ErbB4 cells CUGBP-2, HuR, and RBM3 are all expressed primarily in the nucleus.

\section{ErbB4-induced Cell Survival Requires COX-2 Activity}

We have previously reported that ErbB4 expression protects cells from cytokine-induced apoptosis. ${ }^{13}$ We used the COX-2 inhibitor celecoxib to ask whether ErbB4 induces cell survival through COX-2-dependent mechanisms. After confirming that celecoxib does not nonspecifically interfere with ErbB4 phosphorylation in response to HRG (Figure 6a), we exposed YAMC-Vec and YAMC-ErbB4 cells to a cytotoxic cytokine cocktail (IFN- $\gamma, 150 \mathrm{U} / \mathrm{ml}$; TNF, $100 \mathrm{ng} / \mathrm{ml})$ to stimulate apoptosis $^{30}$ in the presence or absence of celecoxib $(5 \mu \mathrm{M})$. Cell loss was determined using an MTS-based cell count assay. ErbB4 protection from TNF-induced cell death was reversed by the presence of celecoxib (Figure 6b), indicating that COX-2 activity is involved in ErbB4-mediated cell survival. Consistent with these results, shRNA-mediated knockdown of ErbB4 in LIM 2405 human colorectal carcinoma cells, which express high levels of endogenous ErbB4, decreased both steady-state COX-2 protein expression and cell survival in the presence of TNF along with IFN- $\gamma$ (Figure $6 \mathrm{c}$ and $\mathrm{d}$ ). 

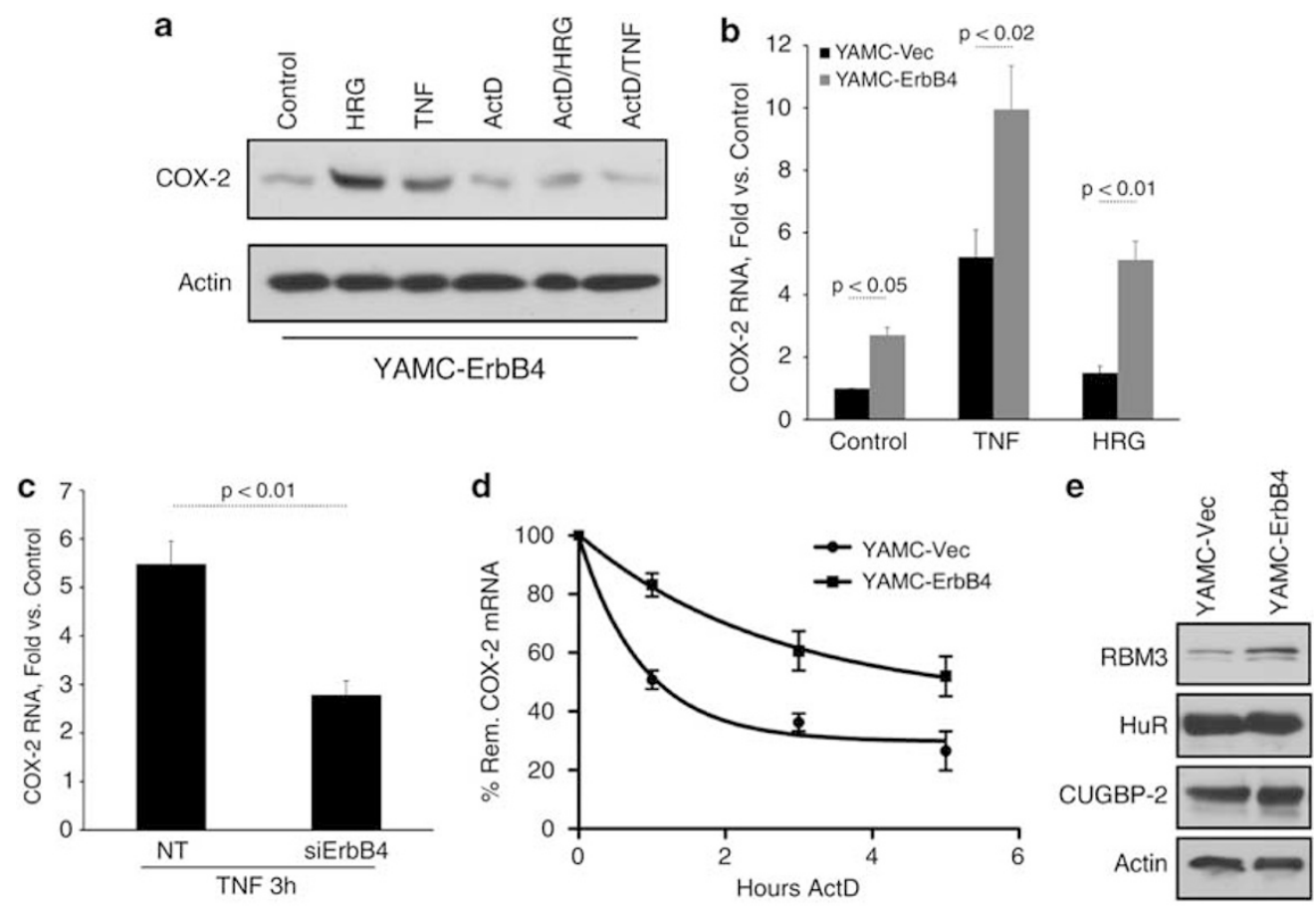

Figure 5 ErbB4 regulates COX-2 mRNA expression. (a) Cells were exposed to HRG or TNF in the presence of actinomycin D (ActD; $10 \mu \mathrm{g} / \mathrm{ml}$ ) and COX-2 protein levels were determined by western blot analysis. (b) JM-b/CYT-2 expressing cells (YAMC-ErbB4) were exposed to tumor necrosis factor (TNF, $100 \mathrm{ng} /$ $\mathrm{ml})$ or HRG $(100 \mathrm{ng} / \mathrm{ml})$ for $3 \mathrm{~h}$; COX-2 mRNA levels were determined by RT-qPCR. (c) YAMC cells were transfected with nontargeting or ErbB4-specific siRNA pools for $72 \mathrm{~h}$. Cells were stimulated with TNF and COX-2 levels were determined by RT-qPCR. (d) Cells were exposed to actinomycin D for indicated times and COX-2 mRNA levels were determined by RT-qPCR. (e) Expression of RBM3, HuR, and CUGBP-2 protein in YAMC-Vec and YAMC-ErbB4 cells was determined by western blot analysis.

\section{ErbB4 Confers Anchorage-Independent Growth through COX-2}

As a loss of appropriate cell death can contribute to cellular transformation and tumorigenesis, we asked whether ErbB4 promotes soft agar colony formation of colon epithelial cells. YAMC-Vec and YAMC-ErbB4 cells were embedded in 0.35\% agar and maintained at permissive conditions for 3 weeks. Three-dimensional cell clusters and colonies were then counted as a measure of transformation. Vector-expressing cells formed only a very few small cell clusters, whereas in contrast ErbB4-expressing cells formed numerous detectable colonies (Figure 7). The ErbB4 ligand HRG enhanced this response, whereas celecoxib blocked ErbB4-stimulated anchorage-independent growth/colony formation either with or without HRG, suggesting that COX-2 activity is required for ErbB4-induced colon epithelial cell transformation.

\section{DISCUSSION}

In this study we show that ErbB4 promotes colon epithelial cell survival in a COX-2-dependent manner. COX-2 induction by ErbB4 was associated with increased mRNA half-life, and required EGFR, Src, and PI 3-kinase activities. These data suggest that elevated ErbB4 expression in inflammatory bowel disease ${ }^{13}$ could contribute to inappropriate cell survival in the inflammatory milieu, thus promoting colitis- associated development of colorectal tumors. Decreased survival of colorectal cancer cells transfected with ErbB4 shRNA constructs ${ }^{14}$ (Figure 6) and the ability of ErbB4expressing cells to form colonies in soft agar in a celecoxibdependent manner (Figure 7) are consistent with this possibility.

In contrast to the fairly clear association of other ErbB family members with tumorigenesis, data in the literature on ErbB4 in cancer are complex. Overexpression has been reported in several cancers including endometrial ${ }^{31}$ and nonsmall cell lung, ${ }^{10,32,33}$ but this correlation is not seen in all tumor types, ${ }^{34-36}$ and studies of breast cancer have yielded contradictory results. ${ }^{37,38}$ In the GI tract the limited available data support a role for ErbB4 in carcinogenesis, with reports noting somatic mutation of the ErbB4 gene in colorectal cancer $^{33}$ or ErbB2/ErbB4 coexpression in late stage tumors. ${ }^{39}$ Leung et $a l^{40}$ recently reported high ErbB4 protein expression in a substantial subset of colorectal tumors examined by immunohistochemical staining. Taken together with these observations, our results linking ErbB4 to expression of COX-2, cell survival and anchorage-independent growth indicate that further investigation into the possible role of ErbB4 in colorectal carcinogenesis is warranted. As it recognizes a uniquely broad subset of EGF-related ligands (HRG/neuregulin growth factors, betacellulin, HB-EGF, and 

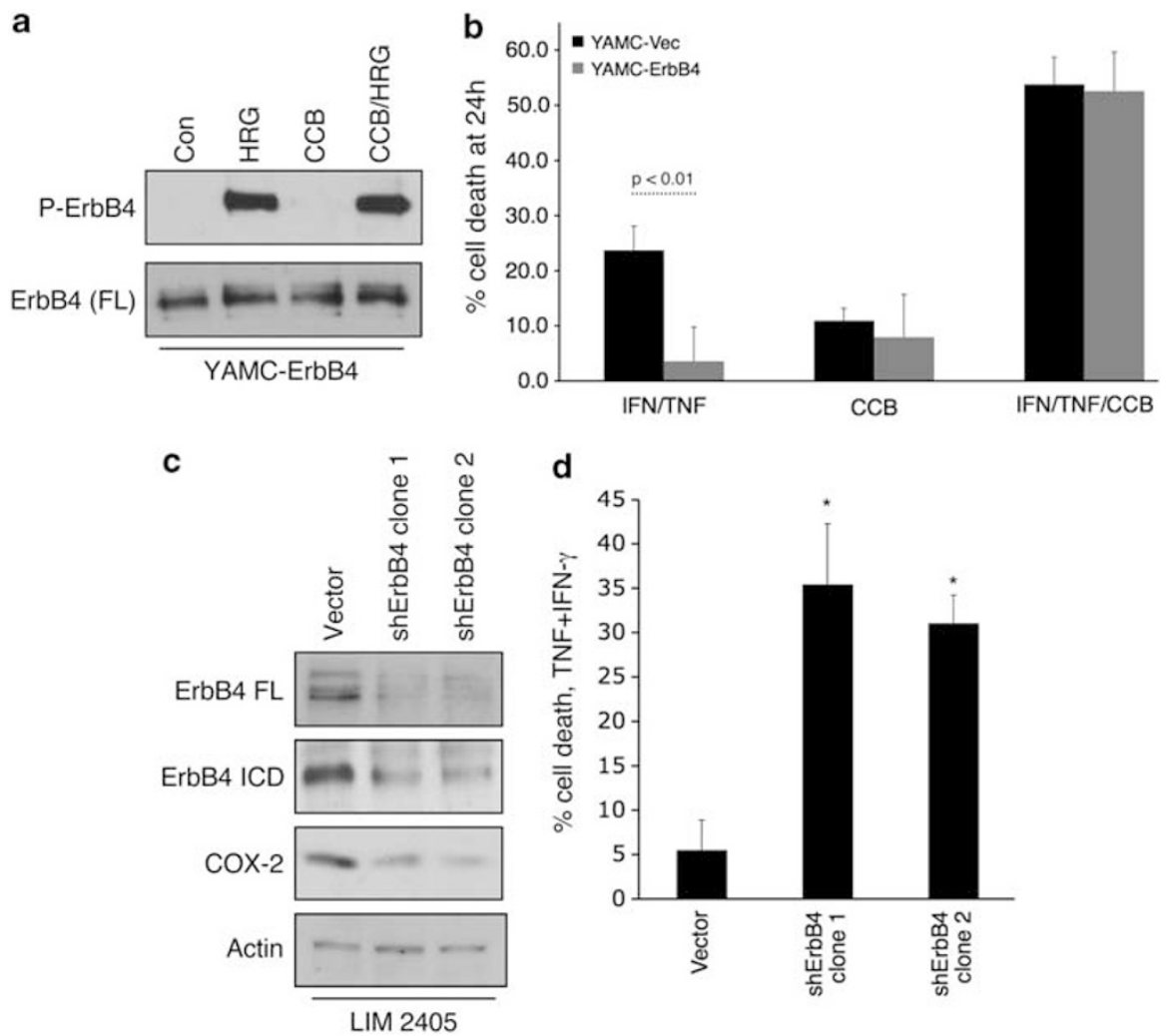

Figure 6 ErbB4-induced cell survival correlates with COX-2 expression. (a) YAMC-ErbB4 cells were exposed to HRG for 15 min with or without the COX-2 inhibitor celecoxib (CCB; $5 \mu \mathrm{M}, 30$ min pretreatment). Cell lysates were prepared and ErbB4 phosphorylation was determined by western blot. (b) YAMC-Vec and YAMC-ErbB4 cells were primed with interferon- $\gamma(150 \mathrm{U} / \mathrm{ml})$ overnight, then exposed to TNF + interferon- $\gamma$ for $24 \mathrm{~h}$ with or without CCB. Cell loss was determined by an MTS-based cell count assay. (c, d) LIM 2405 cells were infected with lentiviral particles to express either control or ErbB4 targeting shRNA constructs and selected with $5 \mu \mathrm{g} / \mathrm{ml}$ puromycin. (c) ErbB4 and COX-2 protein levels were determined by western blot analysis. (d) Cells were exposed to a TNF + IFN $-\gamma$ cocktail for $24 \mathrm{~h}$ and cell loss was determined by MTS cell count. ${ }^{\star} P<0.01$ vs control.

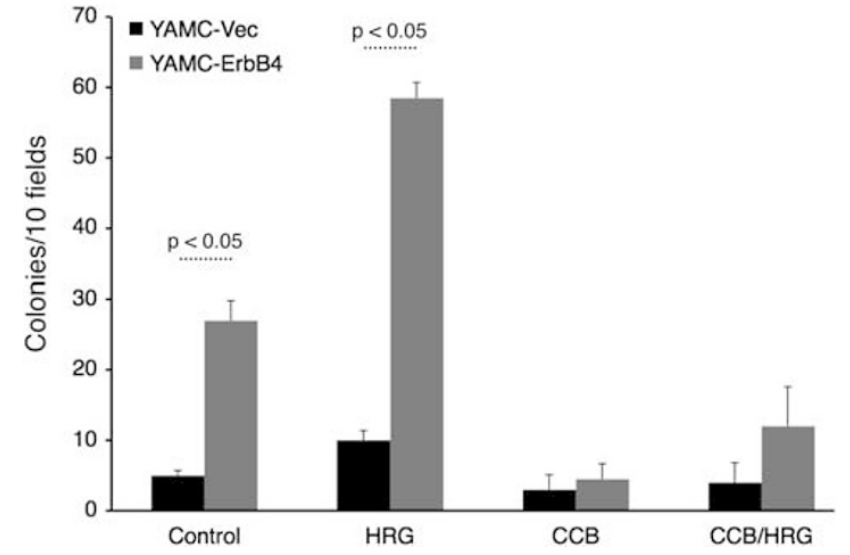

Figure 7 ErbB4 promotes mouse colon epithelial cell transformation. YAMC-Vec and YAMC-ErbB4 were subjected to a soft agar colony-formation assay in the presence or absence of HRG and celecoxib. After 3 weeks colonies ( $>30$ cells) were photographed and counted.

epiregulin) while at the same time binding a more restricted suite of SH2 and PTB domain-containing downstream signaling partners than other ErbBs, ${ }^{12}$ ErbB4 may provide a unique therapeutic target among this receptor family.
The result that COX-2 induced by ErbB4 is dependent on PI 3-kinase signaling (Figure 3) is consistent with our previous data showing that cell survival conferred by ErbB4 overexpression requires this pathway. ${ }^{13}$ Interestingly, this was the case even with expression of CYT-2 isoforms of the molecule, which lack the YTPM motif required for PI 3-kinase binding in NIH 3 T3 cells. $^{6}$ The sensitivity of HRGstimulated Akt activation to Src inhibitors (Figure 3) suggests ErbB4 can stimulate PI 3-kinase indirectly. Furthermore, heterodimerization with EGFR or ErbB3 could result in PI 3-kinase activation and cell survival signaling without the need for YXXM motifs on ErbB4 itself. $^{41}$ For example, a recent report from the Threadgill laboratory suggests that ErbB3/ErbB4 heterodimers are important for survival of HCT116 colon cancer cells; ${ }^{14}$ in a context in which other ErbBs are activated, the CYT-1 YTPM motif on ErbB4 would likely be dispensable for PI 3-kinase activation.

We find that EGFR is rapidly phosphorylated by HRG in YAMC-ErbB4 cells, and conversely EGFR is required for maximal ErbB4 phosphorylation in response to HRG (Figure 4). The most likely interpretation of these data is formation of ErbB4/EGFR heterodimers. However, it is also possible that ErbB4 expression is promoting EGFR transactivation 
through other mechanisms. EGFR can be activated as a result of stimulated ligand release through metalloproteinase activation such as in the T84 colon epithelial cell response to carbachol $^{42}$ or corneal epithelial wound healing accelerated by lysophosphatidic acid. ${ }^{43}$ Ligand-independent intracellular EGFR transactivation pathways have also been shown. ${ }^{44}$ Interestingly, both the ligand-release and intracellular signaling transactivation mechanisms appear to require Src, which directly binds ErbB4-derived phosphopeptides in vitro. ${ }^{12}$ As ErbB4 expression and activation are TNF responsive in colon epithelial cells, ${ }^{13}$ and ErbB4-stimulated COX-2 induction requires Src activity (Figure 4), it is possible that either of these mechanisms are involved in EGFR activation by HRG in YAMC-ErbB4 cells.

ErbB4 expression is typically low in unchallenged colon epithelial cells, but is induced by injury and inflammation, ${ }^{13}$ and promotes cellular survival and transformation. Our data connecting ErbB4 to COX-2 mRNA half-life and thus both basal and stimulated expression levels are novel and consistent with the hypothesis that elevated ErbB signaling during injury and inflammation could promote a tissue environment with elevated EGFR activity, PI 3-kinase/Akt signaling, ${ }^{13}$ and COX-2 expression. This environment would present a favorable niche for the expansion of cells that acquire mutations and subsequent cancer development. Thus, ErbB4 may represent a novel target for intervention in colorectal cancers, particularly those associated with chronic inflammatory diseases. Modulation of ErbB4-mediated signaling, possibly through use of blocking or inactivating antibodies, ${ }^{45,46}$ may provide an avenue to block excessive activation of the PI 3-kinase and COX-2 signaling axes.

\section{ACKNOWLEDGEMENTS}

We thank Jessica Bernard and Lindsay Kuhnhein for expert technical support. This work was supported by NIH Grant K01DK077956 (MRF), American Cancer Society Institutional Research Grant no. IRG-58-009-50 (MRF), a Senior Scientist Award from the Crohn's and Colitis Foundation of America (DBP), NIH Award R01DK056008 (DBP), and the Vanderbilt Digestive Diseases Research Center (VDDRC; NIH Award P30DK058404), including the VDDRC Novel Cell Line Development and Histology core laboratories. The VMC Flow Cytometry Shared Resource is supported by the Vanderbilt Ingram Cancer Center (P30 CA68485) and the VDDRC (DK058404).

\section{DISCLOSURE/CONFLICT OF INTEREST}

The authors declare no conflict of interest.

1. Srinivasan R, Poulsom R, Hurst HC, et al. Expression of the c-erbB-4/ HER4 protein and mRNA in normal human fetal and adult tissues and in a survey of nine solid tumour types. J Pathol 1998;185:236-245.

2. Linggi $B$, Cheng $\mathrm{QC}$, Rao $A R$, et al. The ErbB-4 s80 intracellular domain is a constitutively active tyrosine kinase. Oncogene 2006;25:160-163.

3. Long W, Wagner K-U, Lloyd KCK, et al. Impaired differentiation and lactational failure of Erbb4-deficient mammary glands identify ERBB4 as an obligate mediator of STAT5. Development 2003;130:5257-5268.

4. Vaskovsky A, Lupowitz Z, Erlich S, et al. ErbB-4 activation promotes neurite outgrowth in PC12 cells. J Neurochem 2000;74:979-987.

5. Erlich S, Goldshmit Y, Lupowitz Z, et al. ErbB-4 activation inhibits apoptosis in PC12 cells. Neuroscience 2001;107:353-362.

6. Kainulainen V, Sundvall M, Maatta JA, et al. A natural ErbB4 isoform that does not activate phosphoinositide 3-kinase mediates proliferation but not survival or chemotaxis. J Biol Chem 2000;275: 8641-8649.

7. Tang CK, Goldstein DJ, Payne J, et al. ErbB-4 ribozymes abolish neuregulin-induced mitogenesis. Cancer Res 1998;58:3415-3422.

8. Ni CY, Murphy MP, Golde TE, et al. gamma-Secretase cleavage and nuclear localization of ErbB-4 receptor tyrosine kinase. Science 2001; 294:2179-2181.

9. Srinivasan R, Gillett CE, Barnes DM, et al. Nuclear expression of the c-erbB-4/HER-4 growth factor receptor in invasive breast cancers. Cancer Res 2000;60:1483-1487.

10. Starr A, Greif J, Vexler A, et al. ErbB4 increases the proliferation potential of human lung cancer cells and its blockage can be used as a target for anti-cancer therapy. Int J Cancer 2006;1 19:269-274.

11. Carpenter G. ErbB-4: mechanism of action and biology. Exp Cell Res 2003;284:66-77.

12. Kaushansky A, Gordus A, Budnik BA, et al. System-wide investigation of ErbB4 reveals 19 sites of Tyr phosphorylation that are unusually selective in their recruitment properties. Chem Biol 2008;15:808-817.

13. Frey $\mathrm{MR}$, Edelblum $\mathrm{KL}$, Mullane $\mathrm{MT}$, et al. The ErbB4 growth factor receptor is required for colon epithelial cell survival in the presence of TNF. Gastroenterology 2009;136:217-226.

14. Lee $D, Y u$ M, Lee $E$, et al. Tumor-specific apoptosis caused by deletion of the ERBB3 pseudo-kinase in mouse intestinal epithelium. J Clin Invest 2009;119:2702-2713.

15. Gilroy DW, Colville-Nash PR, Willis D, et al. Inducible cyclooxygenase may have anti-inflammatory properties. Nat Med 1999;5:698-701.

16. Tsujii M, DuBois RN. Alterations in cellular adhesion and apoptosis in epithelial cells overexpressing prostaglandin endoperoxide synthase 2 . Cell 1995;83:493-501.

17. Sun $\mathrm{Y}$, Tang $\mathrm{XM}$, Half $\mathrm{E}$, et al. Cyclooxygenase-2 overexpression reduces apoptotic susceptibility by inhibiting the cytochrome $c$ dependent apoptotic pathway in human colon cancer cells. Cancer Res 2002;62:6323-6328.

18. Singer II, Kawka DW, Schloemann S, et al. Cyclooxygenase 2 is induced in colonic epithelial cells in inflammatory bowel disease. Gastroenterology 1998;115:297-306.

19. Buchanan FG, Holla V, Katkuri S, et al. Targeting cyclooxygenase-2 and the epidermal growth factor receptor for the prevention and treatment of intestinal cancer. Cancer Res 2007;67:9380-9388.

20. Oshima M, Dinchuk JE, Kargman SL, et al. Suppression of intestinal polyposis in Apc delta716 knockout mice by inhibition of cyclooxygenase 2 (COX-2). Cell 1996;87:803-809.

21. Whitehead $\mathrm{RH}$, VanEeden $\mathrm{PE}$, Noble MD, et al. Establishment of conditionally immortalized epithelial cell lines from both colon and small intestine of adult $\mathrm{H}-2 \mathrm{~Kb}$-tsA58 transgenic mice. Proc Natl Acad Sci USA 1993;90:587-591.

22. Whitehead $\mathrm{RH}$, Zhang $\mathrm{HH}$, Hayward IP. Retention of tissue-specific phenotype in a panel of colon carcinoma cell lines: relationship to clinical correlates. Immunol Cell Biol 1992;70(Part 4):227-236.

23. Tong $\mathrm{X}$, Yin $\mathrm{L}$, Joshi $\mathrm{S}$, et al. Cyclooxygenase- 2 regulation in colon cancer cells: modulation of RNA polymerase II elongation by histone deacetylase inhibitors. J Biol Chem 2005;280:15503-15509.

24. Tang $X$, Sun YJ, Half E, et al. Cyclooxygenase-2 overexpression inhibits death receptor 5 expression and confers resistance to tumor necrosis factor-related apoptosis-inducing ligand-induced apoptosis in human colon cancer cells. Cancer Res 2002;62:4903-4908.

25. Parfenova $\mathrm{H}$, Balabanova $\mathrm{L}$, Leffler $\mathrm{CW}$. Posttranslational regulation of cyclooxygenase by tyrosine phosphorylation in cerebral endothelial cells. Am J Physiol 1998;274(1 Part 1):C72-C81.

26. Pinkas-Kramarski $R$, Shelly $M$, Guarino $B C$, et al. ErbB tyrosine kinases and the two neuregulin families constitute a ligand-receptor network. Mol Cell Biol 1998;18:6090-6101.

27. Mukhopadhyay D, Houchen CW, Kennedy S, et al. Coupled mRNA stabilization and translational silencing of cyclooxygenase- 2 by a novel RNA binding protein, CUGBP2. Mol Cell 2003;11:113-126.

28. Sengupta $S$, Jang $B C, W u M T$, et al. The RNA-binding protein HuR regulates the expression of cyclooxygenase-2. J Biol Chem 2003;278: 25227-25233.

29. Sureban SM, Ramalingam S, Natarajan G, et al. Translation regulatory factor RBM3 is a proto-oncogene that prevents mitotic catastrophe. Oncogene 2008;27:4544-4556.

30. Wright K, Kolios G, Westwick J, et al. Cytokine-induced apoptosis in epithelial HT-29 cells is independent of nitric oxide formation. 
Evidence for an interleukin-13-driven phosphatidylinositol 3-kinasedependent survival mechanism. J Biol Chem 1999;274:17193-17201.

31. Srinivasan R, Benton E, McCormick F, et al. Expression of the c-erbB-3/ HER-3 and c-erbB-4/HER-4 growth factor receptors and their ligands, neuregulin-1 alpha, neuregulin-1 beta, and betacellulin, in norma endometrium and endometrial cancer. Clin Cancer Res 1999;5: 2877-2883.

32. al Moustafa $A E$, Alaoui-Jamali $M$, Paterson $J$, et al. Expression of P185erbB-2, P160erbB-3, P180erbB-4, and heregulin alpha in human normal bronchial epithelial and lung cancer cell lines. Anticancer Res 1999;19(1A):481-486.

33. Soung YH, Lee JW, Kim SY, et al. Somatic mutations of the ERBB4 kinase domain in human cancers. Int J Cancer 2006;118:1426-1429.

34. Memon AA, Sorensen BS, Melgard P, et al. Expression of HER3, HER4 and their ligand heregulin-4 is associated with better survival in bladder cancer patients. Br J Cancer 2004;91:2034-2041.

35. Rotterud R, Nesland JM, Berner A, et al. Expression of the epidermal growth factor receptor family in normal and malignant urothelium. BJU Int 2005;95:1344-1350.

36. Edwards J, Traynor P, Munro AF, et al. The role of HER1-HER4 and EGFRvIll in hormone-refractory prostate cancer. Clin Cancer Res 2006;12:123-130.

37. Junttila $T T$, Sundvall $M$, Lundin $M$, et al. Cleavable ErbB4 isoform in estrogen receptor-regulated growth of breast cancer cells. Cancer Res 2005;65:1384-1393.

38. Tovey SM, Witton CJ, Bartlett JM, et al. Outcome and human epidermal growth factor receptor (HER) 1-4 status in invasive breast carcinomas with proliferation indices evaluated by bromodeoxyuridine labelling. Breast Cancer Res 2004;6:R246-R251.

39. Lee JC, Wang ST, Chow NH, et al. Investigation of the prognostic value of coexpressed erbB family members for the survival of colorectal cancer patients after curative surgery. Eur J Cancer 2002;38:1065-1071.

40. Leung SP, Griffith OL, Masoudi H, et al. Clinical utility of type 1 growth factor receptor expression in colon cancer. Am J Surg 2008;195:604-610.

41. Chuu CP, Chen RY, Barkinge JL, et al. Systems-level analysis of ErbB4 signaling in breast cancer: a laboratory to clinical perspective. Mol Cancer Res 2008;6:885-891.

42. McCole DF, Keely SJ, Coffey RJ, et al. Transactivation of the epidermal growth factor receptor in colonic epithelial cells by carbachol requires extracellular release of transforming growth factor-alpha. J Biol Chem 2002;277:42603-42612.

43. Xu KP, Yin J, Yu FS. Lysophosphatidic acid promoting corneal epithelial wound healing by transactivation of epidermal growth factor receptor. Invest Ophthalmol Vis Sci 2007;48:636-643.

44. Caruso R, Pallone F, Fina $D$, et al. Protease-activated receptor-2 activation in gastric cancer cells promotes epidermal growth factor receptor trans-activation and proliferation. Am J Pathol 2006;169: 268-278.

45. Chen X, Levkowitz G, Tzahar E, et al. An immunological approach reveals biological differences between the two NDF/heregulin receptors, ErbB-3 and ErbB-4. J Biol Chem 1996;271:7620-7629.

46. Hollmen M, Maatta JA, Bald L, et al. Suppression of breast cancer cell growth by a monoclonal antibody targeting cleavable ErbB4 isoforms. Oncogene 2009;28:1309-1319. 